\title{
Association between cardiovascular disease and microvascular complications in diabetic patients
}

\author{
Gonzalo Allo Miguel, Elena García Fernández, Juan Carlos Romero Rodríguez, \\ Guillermo Martínez Díaz-Guerra, Federico Hawkins \\ Endocrinology Service, 12 de Octubre University Hospital.
}

\section{Introduction}

Diabetes mellitus is a condition on the increase, carrying a high risk of cardiovascular complications. It is, also, well known that diabetes confers a substantial burden of microvascular disease. Previous studies suggest a link between microvascular and macrovascular events in diabetes. However, just a few studies have investigated whether the presence of previous cardiovascular events is associated with microvascular disease in diabetes patients. So, this is the aim of our study.

Aim

The aim of our study is to evaluate whether the presence of previous cardiovascular events is associated with microvascular disease in diabetic patients.

\section{Patients and methods}

98 diabetic (48 male/50 female) patients were included. Adverse cardiovascular events were collected, including: myocardial infarction, angina, revascularization, etc. Microvascular complications include: retinopathy, nephropathy and peripheral neuropathy. Statistical analysis: Chi-square test was used to compare the existence of microvascular complications between the groups with and without cardiovascular events.

\section{Results}

68 patients with type 2 and 20 with type 1 diabetes with mean age $(52.58 \pm 20.70)$ and mean $\mathrm{HbA} 1 \mathrm{c}(11.21 \pm 2.33 \%)$ were included. 14 patients $(15.56 \%)$ had previous cardiovascular events. The existence of cardiovascular events was significantly correlated with the existence of, al least, one microvascular complication $(p<0.02)$. Also, significant differences between the groups of patients with 0 without cardiovascular disease were found in the rate of neuropathy $(71.43 \%$ and $24.66 \%$ respectively: $p<0.001)$ and in the rate of nephropathy $(57.14 \%$ and $23.61 \%$ respectively; $p<0.001)$. These differences remained significant after adjusting for age, sex and hypertension. There were no significant differences in the rate of retinopathy between both groups.

\begin{tabular}{|c|c|c|c|}
\hline & $\begin{array}{l}\text { Cardiovascular disease - } \\
\qquad(n=84)\end{array}$ & $\begin{array}{l}\text { Cardiovascular disease }+ \\
\qquad(n=14)\end{array}$ & p-value \\
\hline $\begin{array}{l}\text { Peripheral } \\
\text { Neuropathy }\end{array}$ & $18(24.66 \%)$ & $10(71.43 \%)$ & $<0.001$ \\
\hline Nephropathy & $17(23.61 \%)$ & $8(57.14 \%)$ & $<0.001$ \\
\hline $\begin{array}{l}1 \geq \text { Microvascular } \\
\text { complications }\end{array}$ & 7 (9.59\%) & 7 (50.00\%) & 0.02 \\
\hline
\end{tabular}

Table 1. Patients with cardiovascular disease have significant higher rates of microvascular complications: Peripheral neuropathy and nephropathy.

\section{Conclusions}

Our results show significant correlation between the existence of previous cardiovascular events and the existence of one or more microvascular complications. Moreover, we have found that diabetic patients with cardiovascular disease present significant higher rates of nephropathy and neuropathy than diabetic patients without cardiovascular events. Despite the limitations of the study, our results suggest that proper microvascular complications screening seems mandatory in diabetic patients with past cardiovascular events. 